\title{
Index to Volume 133
}

Compiled by William Halliday

Achyranthes japonica, 56

Alaska,

Adak, 49

Aleutian Islands, 49

Amchitka, 49

Attu, 49

Brooks Range, 151

Alberta,

Central, 309

North-Central, 1

Northeastern, 189

Albinism, 113

Alces americanus, 329

Ambystoma laterale, 43

Ammodytes personatus, 144

Amphibians, 43, 101, 193, 196

Anderson, R.B., 1

Annual Reports of OFNC Committees for October 2017-September 2018, 90-95

Ant(s), 309

Araceae, 139

Archaeology, 332

Area(s), Protected, 43, 206, 313

Attaction, Conspecific, 235

Axanthism, 196

Balaenoptera acutorostrata, 144

Barbarea

orthoceras, 118

stricta, 118

verna, 118

Barber-Meyer, S.M., 343

Bat, Hoary, 125

Batch, 325

Bay, Hudson

Bear, Brown, 151

Beaver, American, 332

Behaviour, 235

Hunting, 16

Singing, 28

Swimming, 25

Bellan, L., 305

Biogeography, 156, 206

Bioindicators, 206

Biomass, Below Ground, 364

Bird(s), 20, 28, 167, 235, 301, 305, 352

Cavity-nesting, 352

Paridae, 28
Blaney, C.S., 118

Body, Fat, 34

Bondrup-Nielsen, S., 329

Boudreau, M.J., 329

Boyle, S.P., R. Dillon, J.D. Litzgus, D. Lesbarrères. Desiccation of herpetofauna on roadway exclusion fencing, 43-48

Brassicaceae, 118

Braun, C.E., W.P. Taylor, S.M. Ebbert, L.M. Spitler. Monitoring Rock Ptarmigan (Lagopus muta) populations in the Western Aleutian Islands, Alaska, 49-55

Breeding, 20, 301

Time of, 352

British Columbia, 156

Cormorant Island, 144

Haida Gwaii, 352

Hecate Strait, 263

Kamloops, 28

Kelowna, 28

Kootenays, 221

Williams Lake, 28

Brodo, I., 96

Brunton, D.F. A practical technique for preserving specimens of duckmeal, Wolffia (Araceae), 139143

Bullfrog, American, 43, 196

California, Sierra Nevada Mountains, 34

Callospermophilus lateralis, 34

Cambaridae, 160

Cameron, M.D., 151

Campomizzi, A.J., Z.M. Lebrun-Southcott, K. Richardson. Conspecific cues encourage Barn Swallow (Hirundo rustica erythrogaster) prospecting, but not nesting, at new nesting structures, 235-245

Canis, 16

latrans var., 329

lupus, 60, 343

Cannings, S.G., T.S. Jung, J.H. Skevington, I. Duclos, S. Dar. A reconnaissance survey for Collared Pika (Ochotona collaris) in northern Yukon, $130-135$

Carnivore, 16

Castor canadensis, 332

Catfish, Flathead, 372

Cavity-nesters, 352 
Cavity-nesting, 352

Cedar, K., 160

Cetacean, 144, 263

Chaff-flower, Japanese, 56

Chapman, C.J., C.S. Blaney, D.M. Mazerolle. Wintercresses (Barbarea W.T. Aiton, Brassicaceae) of the Canadian Maritimes, 118-124

Chelydra serpentina, 216

Chickadee, Mountain, 28

Chrosomus neogaeus, 105

Chrysemys picta, 216

Chub,

Creek, 325

Hornyhead, 325

Cipriani, J., 96

Clupea pallasi, 144

Cobb, T.P., 189

Colm, J.E., 372

Colouration, 301

Leucistic, 301

Communication, 28

Competition, Interspecific, 105

Condition, Body, 34

Conductivity, Total Body Electrical, 34

Conservation, 118, 206, 235

Coyotes, Eastern, 329

Crayfish, 160

Cruciferae, 118

Cucumaria frondosa, 113

Cues, Social, 235

Curley, R., D.L. Keenlyside, H.E. Kristmanson, R.L. Dibblee. A review of the historical and current status of American Beaver (Castor canadensis) Cyprinidae, 325 on Prince Edward Island, Canada, 332-342

Dace,

Blacknose, 325

Finescale, 105

Northern Pearl, 325

Dar, S., 130

Decapoda, 160

Den, 1

Deer,

Decoy, 16

White-tailed, 16, 246, 343

DeMaynadier, P.G., 196

Depredation, 60

Design, BACI

Dibblee, R.L., 332

Diet, 151,329

Dillon, R., 43

Dispersal, 136, 332
Distribution, 60, 130, 156, 160, 189, 199, 221

Diversity, Colour, 113

Dorval, H.R., R.T. McMullin. Lichens and allied fungi of Sandbar Lake Provincial Park, Ontario, 206-215

Duck, 167

Duclos, I., 130

Dynamics, Population, 253

Ebbert, S.M., 49

Ecology, Road, 43, 101

Editors' Report for Volume 132 (2018), 298-300

Effects, Ecosystem, 364

Electrofishing, 372

Emydoidea blandingii, 216

Erethizon dorsatum, 25

Excavators, 352

Expansion,

Host, 309

Range, 309

Extension, Range, 56, 156, 160, 189, 199, 221, 309

Fat, 34

Feeding, 144

Fencing, Exclusion, 43

Festarini, A., 305

Fire, 313

Prescribed, 253

Fish, 105, 151, 325, 372

Fishing, 151

Fitness, 301

Floristics, 118

Forbes, G.J., 270

Forest,

Boreal, 1, 206

Great Lakes-St. Lawrence, 206

Formica podzolica, 309

Forsyth, R.G., 313

Forsyth, R.G., J. Kamstra. Roman Snail, Helix pomatia (Mollusca: Helicidae), in Canada, 156-159

Frog(s), 43

American Bull, 43, 196

Gray Tree, 43

Green, 43, 196

Northern Leopard, 43, 193

Wood, 43

Fungi, 206

Gable, D.P., 16

Gable, T.D., D.P. Gable. Wolf (Canis sp.) attacks lifelike deer decoy: insights into how wolves hunt deer? 16-19

Gartersnake, Common, 43 
Gaston, A.J., 352

Gaston, A.J. Birds of Mansel Island, northern Hudson Bay, 20-24

Gaston, A.J., N.G. Pilgrim, V. Pattison. Humpback Whale (Megaptera novaeangliae) observations in Laskeek Bay, western Hecate Strait, in spring and early summer, 1990-2018, 263-269

Gastropod(s), 156, 313

Freshwater, 189

Terrestrial, 221

Glasier, J.R.N., 309

Glass, 305

Glon, M.G., 160

Goldfish, 105

Gosselin, I., 305

Grantham, M., 313

Grieves, L.A., 301

Grit, 305

Ground, Feeding, 144

Ground Squirrel, Golden-mantled, 34

Grouse, Sharp-tailed, 253

Gulo gulo, 1

Habitat, 1, 144

Marine, 136, 144

Nesting, 235

Restoration, 253

Hamel, C.D., 313

Hamel, J.-F., 113

Hanrahan, C., 96

Harpalejeunea molleri subsp. integra, 199

Hart, J., 60

Haughian, S.R., T.H. Neily. Harpalejeunea molleri subsp. integra (R.M. Schuster) Damsholt new to Atlantic Canada, 199-205

Haughton, J., 304

Helix pomatia, 156

Hepatic, 199

Heron, J., 221

Herring, Pacific, 144

Hibernation, 34

Hinchliffe, R.P., C. Tebby, T.P. Cobb. First recorded co-occurrence of Valvata lewisi Currier, 1868 and Valvata lewisi ontariensis Baker, 1931 (Gastropoda: Valvatidae) from Alberta, Canada, with notes on morphometric and genetic variability, 189-192

Hirundo rustica erythrogaster, 235

History, 332

Holothuroidea, 113

Hotspot, 101

Hunter, M.L., Jr., 193

Hybognathus hankinsoni, 325

Hyla versicolor, 43
Illes, C., J.E. Colm, N.E. Mandrak, D.M. Marson. Flathead Catfish (Pylodictis olivaris) reproduction in Canada, 372-380

Impact, Environmental, 305

iNaturalist, 160

Index,

Condition, 34

Urbanization, 28

Information, Public, 235

Insectivore, Aerial, 235

Institute, Alberta Biodiversity Monitoring, 189

Introduction, Intentional, 105

Invasive, 56

Inventory, 221

Isbell, F., 60

Island(s),

Adak, 49

Aleutian Islands, 49

Amchitka, 49

Attu, 49

Campobello, 136

Cormorant, 144

East Sister, 56

Erie, 56

Mansel, 20

Middle, 56

Jokinen, M.E., S.M. Webb, D.L. Manzer, R.B. Anderson. Characteristics of Wolverine (Gulo gulo) dens in the lowland boreal forest of north-central Alberta, 1-15

Joly, K., 151

Jones, C.D., M.G. Glon, K. Cedar, S.M. Paiero, P.D. Pratt, T.J. Preney. First record of Painted Mudbug (Lacunicambarus polychromatus) in Ontario and Canada and the significance of iNaturalist in making new discoveries, $160-166$

Jung, T.S., 130

Jung, T.S. Behaviour of a porcupine (Erethizon dorsatum) swimming across a small boreal stream, $25-27$

Juvenile, 372

Kamstra, J., 156

Kamstra, J. Japanese Chaff-flower, Achyranthes japonica (Amaranthaceae), on the Erie islands, an invasive plant new to Canada, 56-59

Keefe, D.G., R.C. Perry, G.R. McCracken. First records of Finescale Dace (Chrosomus neogaeus) in Newfoundland and Labrador, Canada, 105112

Keenlyside, D.L., 332

Kelt, D.A., 34 
Kinley, T.A. Seasonal movements of White-tailed Deer (Odocoileus virginianus) in the Rocky Mountains of British Columbia, 246-252

Kreuzberg, E., 101

Kristmanson, H.E., 332

Krueger, J., 60

Lacunicambarus polychromatus, 160

Lagopus muta, 49

Lake(s),

Erie, 56, 364

Great, 372

St. Clair, 372

Williams, 28

Lasiurus cinereus, 125

LaZerte, S.E., K.L.D. Marini, H. Slabbekoorn, M.W. Reudink, K.A. Otter. More Mountain Chickadees (Poecile gambeli) sing atypical songs in urban than in rural areas, 28-33

Lebrun-Southcott, Z.M., 235

Lee, D., 305

Lei, C., S.J. Yuckin, R.C. Rooney. Rooting depth and below ground biomass in a freshwater coastal marsh invaded by European Reed (Phragmites australis) compared with remnant uninvaded sites at Long Point, Ontario, 364-371

Lejeuneaceae, 199

Lemnoideae, 139

Lepage, D. Minutes of the $140^{\text {th }}$ Annual Business Meeting (ABM) of the Ottawa Field-Naturalists' Club, 8 January 2019, 88-89

Lepitzki, D., A. Martin. Editors' Report for Volume 132 (2018), 298-300

Lesbarrères, D., 43

Leucism, 301

Leucistic, 301

Lichens, 206

Lindemann, S.B., A.M. O’Brien, T.B. Persons, P.G. DeMaynadier. Axanthism in Green Frogs (Lithobates clamitans) and an American Bullfrog (Lithobates catesbeianus) in Maine, 196198

Lindemann, S.B., D.E. Putnam, M.L. Hunter, Jr., T.B. Persons. Spotless bursni pattern in Northern Leopard Frog (Lithobates pipiens) in Maine, 193-195

Lithobates

catesbeianus, 43, 196

clamitans, 43, 196

pipiens, 43, 193

sylvaticus, 43

Litter, Anthropogenic, 305

Litzgus, J.D., 43
Liverwort, 199

Livestock, 60

Lowlands, 1

Luxilus cornatus, 325

Maine, 193, 196

Mallard, 167

Mammals, 1, 16, 25, 34, 60, 125, 144, 151, 246, 263, $329,332,343$

Management, Prairie, 253

Mandrak, N.E., 372

Manitoba, Southeastern, 313

Manzer, D.L., 1

Margariscus nachtriebi, 325

Marini, K.L.D., 28

Maritimes, 118

Marsh, Coastal, 364

Marson, D.M., 372

Martin, A., 298

Mass, Body, 34

Mazerolle, D.M., 118

McAlpine, D.F. Occurrence of the rare marine littoral millipede, Thalassisobates littoralis (Diplopoda: Nematosomatidae), in Canada, 136-138

McAlpine, D.F., G.J. Forbes. A Tribute to Rudolph Franck Stocek, 1937-2018, 270-275

McCracken, G.R., 105

McCurdy-Adams, H., 216

McLachlan Hamilton, K., 96

McMillan, C.J., 144

McMullin, R.T., 206

Mech, L.D., S.M. Barber-Meyer. Sixty years of Whitetailed Deer (Odocoileus virginianus) yarding in a Gray Wolf (Canis lupus)-deer system, 343351

Mech, L.D., F. Isbell, J. Krueger, J. Hart. Gray Wolf (Canis lupus) recolonization failure: a Minnesota case study, $60-65$

Megaptera novaeangliae, 263

Mercier, A., 113

Metal, 305

Migration, 343

Millipede, Marine, 136

Minnesota, 60

Northeastern, 343

Minnow(s), 325

Brassy, 325

Minutes of the $140^{\text {th }}$ Annual Business Meeting (ABM) of the Ottawa Field-Naturalists' Club, 8 January $2019,88-89$

Mitigation, Road-effect, 43, 216

Mollusca, 156, 189, 221, 313

Monitoring, Population, 49 
Montgomery, E.M., T. Small, J.-F. Hamel, A. Mercier. Albinism in Orange-footed Sea Cucumber (Cucumaria frondosa) in Newfoundland, 113-117

Moore, K., 352

Moose, 329

Morningstar, D., A. Sandilands. Summer movements of a radio-tagged Hoary Bat (Lasiurus cinereus) captured in southwestern Ontario, 125-129

Mortality, 43, 167

Motus, 125

Mountains, 151

Nahoni, 130

Richardson, 130

Sierra Nevada, 34

Movement,

Seasonal, 125, 246, 263

Summer, 125

Mudbug, Paintedhand, 160

Muntz, E.M., 329

Murphy, R.K., K.A. Smith. Sharp-tailed Grouse (Tympanuchus phasianellus) population dynamics and restoration of fire-dependent northern mixed-grass prairie, 253-262

Neily, T.H., 199

Nesting, 235, 305

Cavity, 352

Nestling, 305

New Brunswick, Campobello Island, 136

Newfoundland and Labrador, 113

Exploits River, 105

Newt, Eastern, 101

Nicolai, A., R.G. Forsyth, M. Grantham, C.D. Hamel. Tall grass prairie ecosystem management-a gastropod perspective, 313-324

Nocomis biguttatus, 325

North Atlantic, 113

North Dakota, 253

Notophthalmus viridescens, 101

Nova Scotia, 199

Cape Breton, 329

Nunavut, Mansel Island, 20

O’Brien, A.M., 196

Occurrence, Seasonal, 263

Oceanography, 263

Ochotona collaris, 130

Odocoileus virginianus, 16, 246, 343

Oncorhynchus spp., 151

Ontario, 156

Eastern, 216, 305
Killarney, 16

Lake Erie, 56

Long Point, 364

Northwestern, 206

Southern, 43, 235, 301, 325

Southwestern, 125, 160, 364, 372

Ornithology, 20, 28, 167, 235, 301, 305, 352

Otter, K.A., 28

Ovaska, K., L. Sopuck, J. Heron. Survey for terrestrial gastropods in the Kootenay region of British Columbia, with new records and range extensions, 221-234

Paiero, S.M., 160

Paper, 305

Parasitism, Dulotic, 309

Park,

Cape Breton Highlands National, 329

Dàadzàii Vàn Territorial, 130

Gates of the Arctic National Park and Preserve, 151

Gatineau, 101

Herring Cove Provincial, 136

Jarvis Bay Provincial, 309

Killarney Provincial, 16

Kootenay National, 246

Ni'iinlii Njik (Fishing Branch) Territorial, 130

Presqu'ile Provincial, 43

Sandbar Lake Provincial, 206

Pattison, V., 263

Perry, R.C., 105

Persons, T.B., 193, 196

Phragmites australis, 364

Piercey, R.S., 144

Pika, Collared, 130

Pilgrim, N.G., 263

Pilgrim, N.G., J.L. Smith, K. Moore, A.J. Gaston. Nest site characteristics of cavity-nesting birds on a small island, in Haida Gwaii, British Columbia, Canada, 352-363

Plant(s), 56, 118, 139, 199

Plastic, 305

Poecile gambeli, 28

Polyergus bicolor, 309

Population, 49, 253, 263

Porcupine, North American, 25

Power, J.W.B., M.J. Boudreau, E.M. Muntz, S. Bondrup-Nielsen. High reliance on a diet of Moose (Alces americanus) by Eastern Coyotes (Canis latrans var.) in Cape Breton Highlands National Park, Nova Scotia, Canada, 329-331 
Prairie,

Northern Mixed-grass, 253

Tall Grass, 313

Pratt, P.D., 160

Predation, 16, 343

Predator-prey, 16

Preney, T.J., 160

Preparation, Herbarium Specimen, 139

Prince Edward Island, 332

Prospecting, 235

Ptarmigan, Rock, 49

Putnam, D.E., 193

Pylodictis olivaris, 372

Quebec, Gatineau, 101

Quinn, N.W.S. Batch spawning in five species of minnows (Cyprinidae) from Ontario, Canada, 325328

Range,

Distributional, 130

Summer, 246

Winter, 246

Recolonization, 60

Record,

New, 118

New Distribution, 160, 221

New Provincial, 156, 160

Reed, European, 364

Reintroduction, 60

Relations, Predator-prey, 343

Reproduction, 372

Reptiles, 43, 216

Residuals, Mass-length, 34

Restoration, Habitat, 235

Reudink, M.W., 28

Review, 332

Rhinichthys artatulus, 325

Rhizomes, 364

Richardson, K., 235

River,

Klondike, 25

Thames, 372

Robin, American, 301

Rood, S.B., A. Willcocks. Duckling mortality at a river weir, $167-171$

Rooney, R.C., 364

Roots, 364

Salamander, 101

Blue-spotted, 43

Salmon, 151

Sand Lance, Pacific, 144
Sandilands, A., 125

Scat, 329

Sea Cucumber, Orange-footed, 113

Seburn, D.C., E. Kreuzberg, L. Viau. Roadkill of Eastern Newts (Notophthalmus viridescens) in a protected area in Quebec, 101-104

Seburn, D.C., H. McCurdy-Adams. Do turtle warning signs reduce roadkill? 216-220

Selection, Sexual, 301

Semotilus atromaculatus, 325

Shiner, Common, 325

Signs,

Turtle Warning, 216

Wildlife, 216

Site, Nest, 352

Skevington, J.H., 130

Slabbekoorn, H., 28

Slugs, Terrestrial, 313

Small, T., 113

Smith, J.L., 352

Smith, K.A., 253

Snail(s),

Freshwater, 189, 313

Roman, 156

Terrestrial, 156, 221, 313

Snakes, 43

Common Garter, 43

Snow, 1

Songs, Atypical, 28

Sopuck, L., 221

Sorum, M.S., K. Joly, M.D. Cameron. Use of salmon (Oncorhynchus spp.) by Brown Bears (Ursus arctos) in an Arctic, interior, montane environment, 151-155

Sosiak, C.E., M. West, J.R.N. Glasier. First record and new host record of the obligate dulotic ant, Polyergus bicolor (Hymenoptera: Formicidae), in Alberta, Canada, 309-312

Spawning, Batch, 325

Species,

Anthropochorus, 136

Exotic, 105

Invasive, 56, 364

Rare, 313

Spitler, L.M., 49

Squirrel,

Golden-mantled Ground, 34

Ground, 34

Status, Population, 49, 332

Storage, 139

Strait, Hecate, 263

Structures, Nesting, 235

Stuart, M., 305 
Survey,

Acoustic, 28

Aerial, 130

Camera Trap, 1

Electrofishing, 372

Fyke Net, 105

Gill Net, 105

Hoop Net, 372

Live Trap, 1

Minnow Pot, 105, 325

Passive Acoustic, 28

Point Count, 43

Road, 101

Scat, 329

Trammel Net, 372

Transect, 329, 352

Visual, 43, 101, 130, 144, 151, 206, 263, 329, 352

Swallow,

Barn, 235

Tree, 305

Tachycineta bicolor, 305

Taylor, W.P., 49

Tebby, C., 189

Telemetry,

GPS, 1

Radio, 125, 343

Thalassisobates littoralis, 136

Thamnophis sirtalis, 43

Thorn, R.G., 301

Towers, J.R., C.J. McMillan, R.S. Piercey. Sighting rates and prey of Minke Whales (Balaenoptera acutorostrata) and other cetaceans off Cormorant Island, British Columbia, 144-150

Treefrog, Gray, 43

Trees, Wildlife, 352

Trends, Population, 263

Tribute, 270

Turdus migratorius, 301

Turtle, 216

Blanding's, 216

Painted, 216

Snapping, 216

Tympanuchus phasianellus, 253

Urbanization, 28

Ursus arctos, 151

Use, Habitat, 144
Valvata, 189

lewisi, 189

lewisi ontariensis, 189

Valvatidae, 189

Van Vuren, D.H., 34

Variant,

Blue Colour, 196

Pattern, 193

Viau, L., 101

Vocalization, 28

Walsh, S., J. Haughton, L. Bellan, I. Gosselin, A. Festarini, D. Lee, M. Stuart. Occurrence of anthropogenic litter in nestling Tree Swallows (Tachycineta bicolor), 305-308

Waterfowl, 167

Watson, J.A., 34

Webb, S.M., 1

Weir, River, 167

Wells, C.P., J.A. Watson, D.A. Kelt, D.H. Van Vuren. Body mass as an estimate of female body condition in a hibernating small mammal, 34-42

West, M., 309

Wetlands, 364

Whale,

Humpback, 263

Minke, 144

Willcocks, A., 167

Wintercresses, 118

Wolf, 16

Gray, 60, 343

Wolffia, 139

Wolverine, 1

Yard, Deer, 343

Yarding, 343

Young-of-year, 372

Yukin, S.J., 364

Yukon, Klondike River, 25

Zitani, N.M., L.A. Grieves, R.G. Thorn. A successfully breeding, partially leucistic American Robin (Turdus migratorius), 301-304

Zurbrigg, E., I. Brodo, J. Cipriani, C. Hanrahan, K. McLachlan Hamilton. The Ottawa Field-Naturalists' Club Awards for 2018, presented February 2019, 96-99 


\section{Index to Book Review}

\section{Botany}

Brunton, D.F. "Flora of Florida Volume 6 (Dicotyledons, Convolvulaceae through Paulowniaceae)" by R.P. Wunderlin, B.F. Hansen, and A.R. Franck, 2019, 70

Brunton, D.F., M.J. Oldham. "Michigan Ferns \& Lycophytes: A Guide to Species of the Great Lakes Region" by D.D. Palmer, 2018, 68-69

Clarkin, O. "Identification of Trees and Shrubs in Winter Using Buds and Twigs" by Bernd Schulz, 2018, 71-72

Cray, H.A. "Seaweed Chronicles" by Susan Hand Shetterly, 2018, 276

Crins, W.J. "Sedges of the Northern Forest: A Photographic Guide" by Jerry Jenkins, 2019, $172-173$

\section{Climate Change}

Brooks, R. "The Uninhabitable Earth: Life After Warming" by David Wallace-Wells, 2019, 6668

\section{Entomology}

Cottam, B. "Butterflies: Their Natural History and Diversity. Second Edition” by Ronald Orenstein, Photography by Thomas Marent, 2020, 383

Cray, H.A. "Buzz, Sting, Bite: Why We Need Insects" by Anne Sverdrup-Thygeson, 2019, 381-382

Lauff, R. "Field Guide to Flower Flies of Northeastern North America" by Jeffrey H. Skevington, Michelle M. Locke, Andrew D. Young, Kevin Moran, William J. Crins, and Stephen A. Marshall, 2019, 73

\section{Herpetology}

Seburn, D. "The Field Herping Guide: Finding Amphibians and Reptiles in the Wild" by Mike Pingleton and Joshua Holbrook, 2019, 384

\section{Ornithology}

Clark, H.O., Jr. "Feed the Birds: Attract and Identify 196 Common North American Birds" by Chris Earley, 2019, 279-280

Curry, B. "The Handbook of Bird Families" by Jonathan Elphick, 2018, 74-75

Foster, R.F. “Ospreys: The Revival of a Global Raptor" by Alan F. Poole, 2019, 175

Gaston, T. "Gulls” by John C. Coulson, 2019, 174
Gaston, T. "Gulls of the World: A Photographic Guide" by Klaus Malling Olsen, 2018, 385

Lein, M.R. "Birds of Saskatchewan" by Alan R. Smith, C. Stuart Houston, and J. Frank Roy, 2019, 277-278

\section{Other}

Bocking, E. "Surviving Global Warming: Why Eliminating Greenhouse Gases Isn't Enough" by Roger A. Sedjo, 2019, 390-391

Burke, T. "How to Give Up Plastic: A Guide to Changing the World, One Plastic Bottle at a Time" by Will McCallum, 2018, 283

Burke, T. "Plastic Soup: An Atlas of Ocean Pollution" by Michiel Roscam Abbing, 2019, 285

Cottam, B. New Titles, 80-83, 184-186, 289-293, 394-397

Cottam, B. “To Speak for the Trees: My Life's Journey from Ancient Celtic Wisdom to a Healing Vision of the Forest" by Diana BeresfordKroeger, 2019, 181-182

Cottam, B. "The Overstory: A Novel" by Richard Powers, 2018, 183

Cottam, B. "Mama's Last Hug: Animal and Human Emotions" by Frans de Waal, 2019, 286-287

Gaston, T. "North Pole: Nature and Culture" by Michael Bravo, 2019, 288

Hartzell, S.M. "The Environment: A History of the Idea" by Paul Warde, Libby Robin, and Sverker Sörlin, 2018, 76

Hunter, M.L., Jr. "The Great Himalayan National Park: The Struggle to Save the Western Himalayas" by Sanjeeva Pandey and Anthony J. Gaston, 2019, 77-78

Pazhoohi, F. "How to Walk on Water and Climb up Walls: Animal Movement and the Robots of the Future" by David L. Hu, 2018, 392

Sander-Regier, R. "Frog Pond Philosophy: Essays on the Relationship Between Humans and Nature" by Strachan Donnelley, 2018, 393

\section{Zoology}

Clark, H.O., Jr. "The Flying Zoo: Birds, Parasites, and the World They Share" by Michael Stock, 2019, 386-387

Cottam, B. "Mammal Tracks and Sign: A Guide to North American Species. Second Edition" by Mark Elbroch with contributions by Casey McFarland, 2019, 178-179

Cray, H.A. "Bats: An Illustrated Guide to All Spe- 
cies" by Marianne Taylor, Photography by Merlin D. Tuttle, 2018, 177

Gibson, J.F. "The New Beachcomber's Guide to the Pacific Northwest” by J. Duane, 2019, 176

Gibson, J.F. "A Field Guide to Marine Life of the Protected Waters of the Salish Sea" by Rick M. Harbo, 2019, 176

Gibson, J.F. "A Field Guide to Marine Life of the Outer Coasts of the Salish Sea and Beyond" by Rick M. Harbo, 2019, 176

Halliday, W.D. "The North Atlantic Right Whale: Disappearing Giants. Revised and Updated Edition" by Scott Kraus, Marilyn Marx, Heather Pettis, Amy Knowlton, and Kenneth Mallory, 2019, 281
Halliday, W.D. “Orca: The Whale Called Killer. Fifth Edition" by Erich Hoyt, 2019, 388

Lauff, R. "Mammals of Prince Edward Island and Adjacent Marine Waters" by Rosemary Curley, Donald F. McAlpine, Dan McAskill, Kim Riehl, and Pierre-Yves Daoust, 2019, 389

Way, J. "Return of the Wolf: Conflict and Coexistence" by Paula Wild, 2018, 78-79

Way, J. "The Rise of Wolf 8: Witnessing the Triumph of Yellowstone's Underdog" by Rick McIntyre, 2019, 180-181

Way, J. "Yellowstone Cougars: Ecology Before and During Wolf Restoration" by Toni K. Ruth, Polly C. Buotte, and Maurice G., 2019, 282283 